\title{
Edge plasma measurements near the minimum-B anchor cell of GAMMA10 using Langmuir probe and calorimeter arrays
}

\author{
Y. Nakashima, ${ }^{\text {a) }}$ M. K. Islam, T. Natori, I. Katanuma, T. Ohki, K. Watanabe, \\ Y. Higashizono, and T. Cho \\ Plasma Research Center, University of Tsukuba, Tsukuba, Ibaraki 305-8577, Japan \\ Y. Ishimoto \\ Japan Atomic Energy Research Institute, Naka Fusion Research Establishment, Nakamachi, Naka-gun, \\ Ibaraki 311-0193, Japan
}

(Presented on 22 April 2004; published 20 October 2004)

\begin{abstract}
Edge plasma diagnostic system equipped in minimum-B anchor cell of the GAMMA10 tandem mirror is described together with the measured results. Several sets of the diagnostic element consisting of a Langmuir probe and a calorimeter are placed in array on the conducting plates installed in the east anchor cell. This diagnostic system is designed to be capable of precisely investigating the spatial dependence of the edge plasma parameters. In typical ion cyclotron range of frequency-heated plasmas, detailed behavior of the edge plasma in the anchor transition regions has been measured by using this system. From these measurements a significant asymmetry of spatial profile was observed in the floating potentials. Heat flow profiles also showed the similar dependence to the probe signals. In this region there exists a strong gradient of the magnetic field strength and the direction of the observed shift is found to be consistent with that of gradient B drift for ions. (C) 2004 American Institute of Physics. [DOI: 10.1063/1.1787577]
\end{abstract}

\section{INTRODUCTION}

Tandem mirror devices are inevitably to be equipped with magnetohydrodynamic (MHD) stabilization function such as minimum-B configuration. In the GAMMA10 tandem mirror ${ }^{1,2}$ two minimum-B anchor cells, which consist of baseball coil and racetrack coil, are installed at both ends of the central cell. A series of investigations have been performed on the anchor cell plasmas from the viewpoint of MHD stability. ${ }^{3,4}$ The anchor cell contains two transition regions, such as an inner-transition region connecting the magnetic field line from the central cell to the anchor cell and an outer-transition region located between the anchor midplane and the plug/barrier cell. In these transition regions, a cross section of plasmas is elliptically elongated under a nonaxisymmetric structure of magnetic field configuration. This region also contains a strong gradient- $\mathrm{B}$ structure where the magnetic field line of the minimum-B region is connected with the axisymmetric field line of the central and plug/ barrier region. ${ }^{5}$ From such circumstances, there may be provided significant characteristics to the plasmas in this region. However, behavior of edge plasmas in the anchor transition regions had not been investigated sufficiently, since the accessibility was limited due to the magnetic coil system surrounding the plasma.

Several years ago four sets of conducting plates were installed in the anchor transition regions, which enabled us to install an extensive scale of the diagnostic system and to carry out the edge plasma measurements in this region. ${ }^{6-9}$

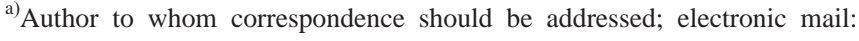
nakashma@prc.tsukuba.ac.jp
}

The objective of this study is to build up a diagnostic system using Langmuir probe and calorimeter arrays installed on the anchor conducting plates and to investigate the edge plasma behavior in the nonaxisymmetric transition region.

\section{EXPERIMENTAL APPARATUS}

Figure 1 shows the schematic view of the GAMMA10 tandem mirror and the detail of an anchor cell together with the location of the diagnostics used for this study. GAMMA10 is a tandem mirror device, which consists of an axisymmetric central-mirror cell, two anchor cells with minimum-B field, and two plug/barrier cells with axisymmetric mirrors. ${ }^{1,2}$ The total length of GAMMA10 is $27 \mathrm{~m}$ and the magnetic strength in the central cell is normally $0.43 \mathrm{~T}$ and varied from 0.3 to $0.57 \mathrm{~T}$. Both ends of the central cell are connected to the anchor cells through the mirror throat regions.

Initial plasma is injected from both ends by plasma guns, and then plasma is built up with ion cyclotron range of frequency (ICRF) wave together with gas puffing. One of the ICRF waves (RF1) is mainly used for MHD stabilization and excited by so-called NAGOYA-type III antenna. Central-cell ions are heated by another ICRF wave (RF2) using a pair of the double half-turn antennas located near the ends of the central cell. End-loss particles from the central-cell plasma are confined by the plug potential produced with electron cyclotron heating $(\mathrm{ECH})$.

The schematic view of the anchor conducting plates and magnetic flux tube in the east anchor cell is illustrated in Fig. 1(b). The conducting plates are made of stainless steel with $2 \mathrm{~mm}$ thickness and installed closely to the plasma surface 


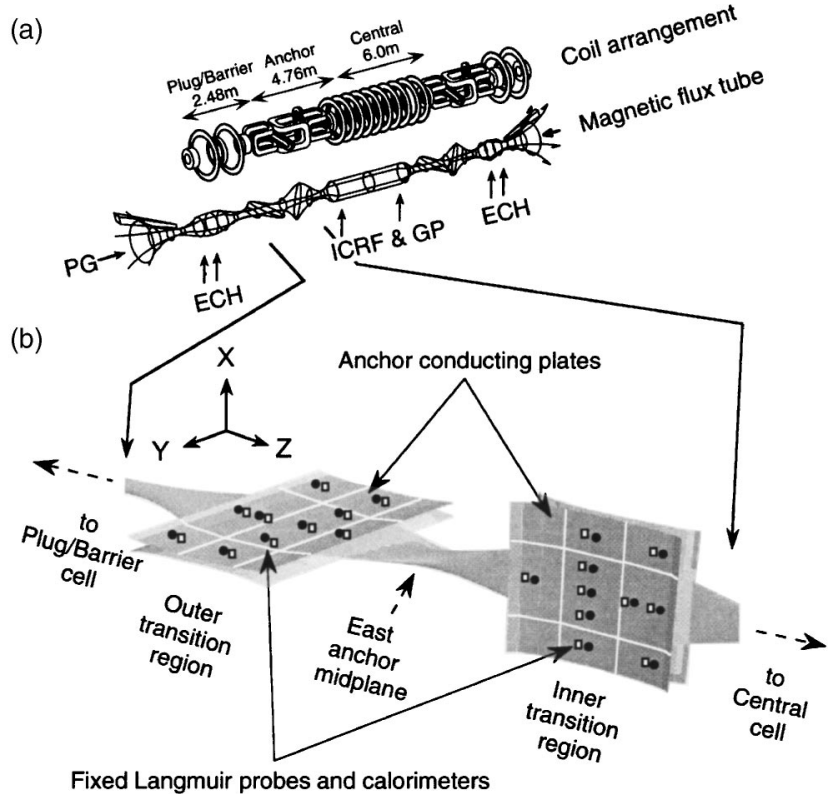

FIG. 1. Schematic view of GAMMA10. (a) Coil arrangement, shape of the magnetic flux tube, and location of heating systems, (b) magnetic flux tube in the east anchor cell, anchor conducting plates and location of diagnostic equipments.

along such a flat magnetic flux tube in the anchor transition regions of both anchor cells. The conducting plates, which are installed in parallel with the longer axis of the elliptic plasma cross section, are separated into three pieces in axial and azimuthal directions, respectively. Each plate is capable of changing the potential in floating and grounded conditions individually, and also can be biased by using external power supply. In this experiment, all the conducting plates were kept floating. On the plates of the east anchor-transition regions, several sets of diagnostic element consisting of a Langmuir probe and a calorimeter are located in array in order to investigate the behavior of the edge plasmas.

Figure 2 shows the detail of the diagnostic element and its data acquisition system. Tips of the Langmuir probe made of tungsten wire, which are aligned in axial ( $z$ axis) and azimuthal ( $x, y$ axis) directions as shown in Fig. 1(b), collect the spatial information of edge plasmas $2 \mathrm{~mm}$ apart from the surface of the conducting plates. The signal from the probe array is transferred to a CAMAC system by way of an iso-

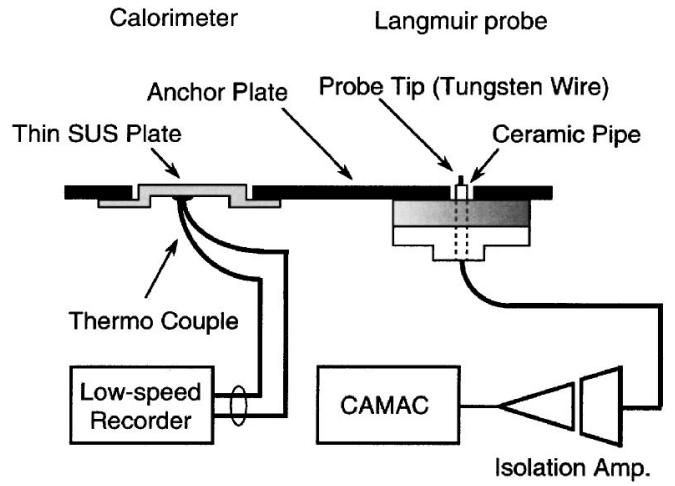

FIG. 2. Schematic view of calorimeter and Langmuir probe and data acquisition systems.
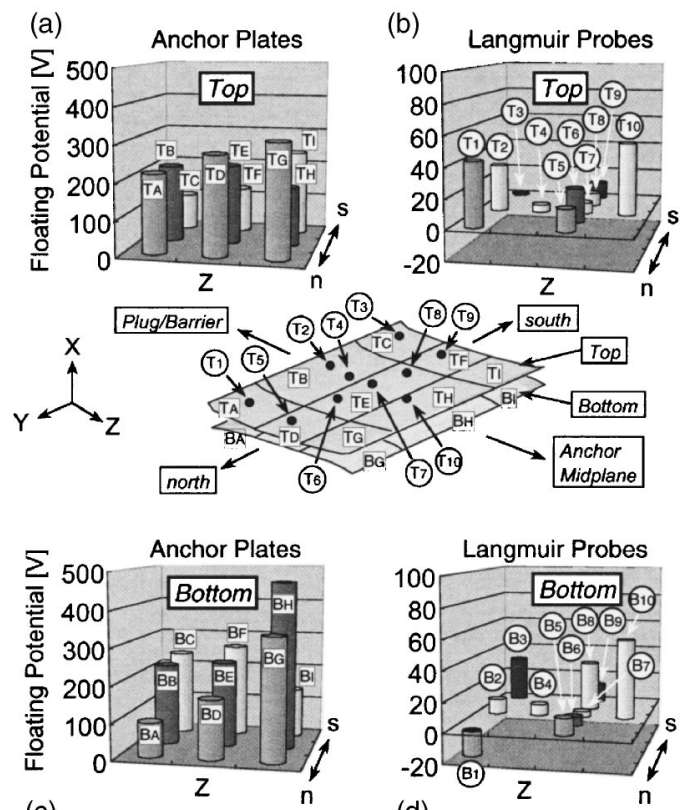

(c)

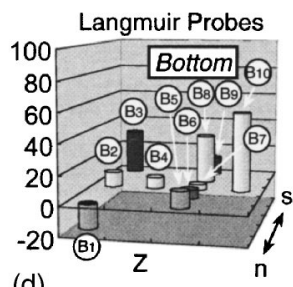

(d)

FIG. 3. Spatial profile of floating potentials of anchor plates and of Langmuir probe array measured at the outer-transition region of the east anchor cell. Characters enclosed by squares represent the number of anchor plates. Those enclosed by circles correspond to the location of Langmuir probes.

lation amplifier and is processed into temporal and spatial distributions with a workstation. The calorimeter element made of thermocouple (copper-constantan) welded on a thin SUS plate is placed close to the Langmuir probe. The heat flow from the edge plasma is detected as a temperature rise of the thin plate measured with the thermocouple and the signal is taken into a low-speed analog recorder as an averaged value during a plasma discharge.

\section{EXPERIMENTAL RESULTS AND DISCUSSION}

In typical ICRF-heated hot-ion-mode ${ }^{10}$ plasmas, edge plasma measurements have been carried out in the anchor cell using this diagnostic system. Figure 3 shows the floating potentials of the conducting plates in the east outer-transition region and those measured with the probe array on the same plates. These results are obtained in the period of time without a confining potential by ECH and the plasma is sustained with only ICRF waves. As shown in Figs. 3(a) and 3(c), all the conducting plates reveal positive floating potentials, which indicates that ion influx to the plate is predominant for the charge balance. On the top plate, particularly in Fig. 3(a), the floating potential increases from the south to the north (from $T_{C}$ to $T_{A}$ and from $T_{F}$ to $T_{D}$ ). This tendency becomes remarkable according to the increase of the distance from the anchor midplane. Bottom plates, on the other hand, show the inverse dependence in $y$ direction (from $\mathrm{B}_{\mathrm{A}}$ to $\mathrm{B}_{\mathrm{C}}$ and from $B_{D}$ to $B_{F}$ ). In the potential profile obtained from the probe, as shown in Figs. 3(b) and 3(d), such dependence is much more enhanced. Especially in the probe array far away from the anchor midplane, observed potential shows the negative value in the north side $\left(\mathrm{B}_{1}\right)$, which implies that the electron 

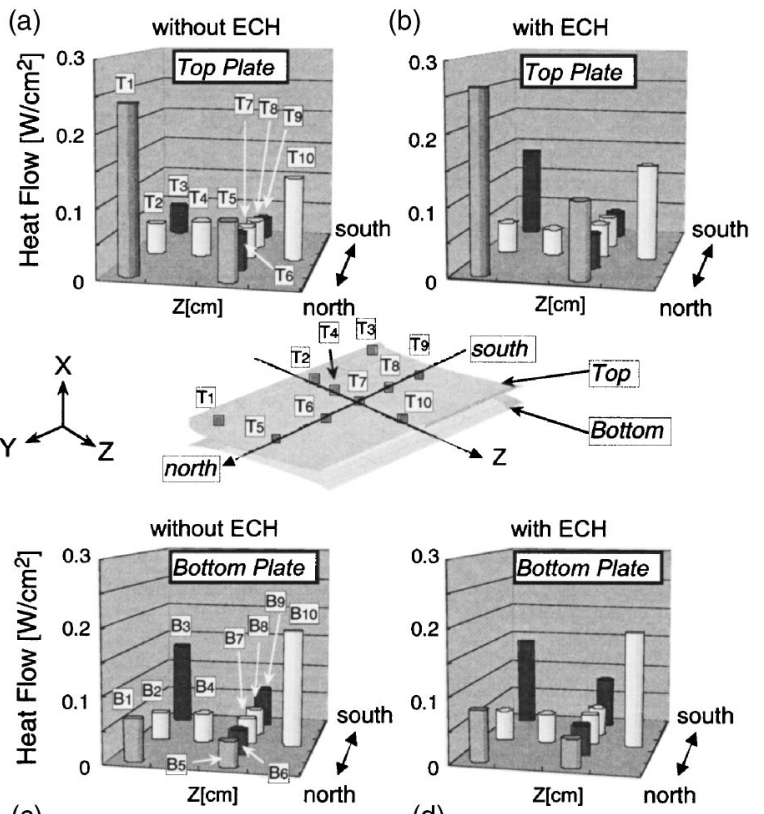

(c)

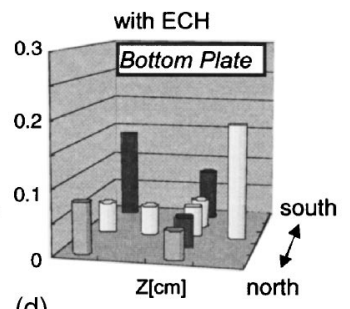

(d)

FIG. 4. Spatial profile of heat flow from the edge plasma measured with the calorimeter array. Without ECH cases: (a), (c). With ECH cases: (b), (d). Characters enclosed by squares correspond to the location of Langmuir probes.

current prevails over the decreasing ion current. The probe located at the south end on the top plate shows the same result $\left(\mathrm{T}_{3}\right)$.

Figure 4 shows the results of calorimeter measurements on the same conducting plates obtained in the similar plasma shots as shown in Fig. 3. As shown in Figs. 4(a) and 4(c), observed heat flow intensities into the top plates increase from the south $\left(\mathrm{T}_{3}, \mathrm{~T}_{9}\right)$ to the north $\left(\mathrm{T}_{1}, \mathrm{~T}_{5}\right)$ and those into the bottom plates show the inverse tendency (from $B_{1}$ to $B_{3}$ and from $\mathrm{B}_{5}$ to $\mathrm{B}_{9}$ ). These results give a good agreement with the results of the floating potential measurement, which shows that the heat flow is dominated by ion flux. Although the results presented in Figs. 4(b) and 4(d) are obtained in the plasma shot with the potential confinement (with ECH), there is no remarkable difference between both cases. This means that the electron heating due to ECH does not affect the heat flux. From the above results described, it is revealed that the observed asymmetry is enhanced in the region far from the anchor midplane where the cross section of the plasma is elongated flatly in a strong gradient of the magnetic field. ${ }^{6}$ Observed area with high floating potential and high heat-flow density corresponds to the direction of gradient-B drift of plasma ions. ${ }^{9}$ Since the location of the main plates corresponding to the magnetic flux tube is sufficiently apart from the plasma boundary $\left(r_{\text {plate }} \geqslant 1.5 r_{\text {plasma }}\right)$, it is difficult for electrons to reach the plates across the magnetic field lines. From these results, the asymmetry of the edge plasma parameters measured in the outer-transition region is ascribed to the effect of ion drift motion in this region. These results showed that the present diagnostic system enabled us to understand the detailed behavior of edge plasmas in the nonaxisymmetric anchor transition region.

\section{ACKNOWLEDGMENTS}

The authors would like to acknowledge the members of the GAMMA10 group, University of Tsukuba, for their collaboration in the experiments.

${ }^{1}$ M. Inutake et al., Phys. Rev. Lett. 55, 939 (1985).

${ }^{2} \mathrm{~S}$. Miyoshi et al., Proceedings of the 13th International Conference on Plasma Phys. and Controlled Nucl. Fusion Research, Washington D.C., 1990 (IAEA, Vienna, 1991), Vol. 2, p. 539.

${ }^{3}$ M. Ichimura et al., Nucl. Fusion 28, 799 (1988).

${ }^{4}$ M. Inutake et al., Trans Fusion Technol. 27, 409 (1995).

${ }^{5}$ M. K. Islam et al., J. Phys. Soc. Jpn. 69, 2493 (2000).

${ }^{6}$ Y. Nakashima et al., J. Plasma Fusion Res. 75, 1211 (1999).

${ }^{7}$ Y. Nakashima et al., J. Nucl. Mater. 290-293, 683 (2001).

${ }^{8}$ Y. Nakashima et al., Proceedings of the International Conference on Open Magnetic Systems for Plasma Confinement, Tsukuba, Japan, 3-6 July 2000, Trans. Fusion Technol. 39, 139 (2001).

${ }^{9}$ Y. Nakashima et al., J. Plasma Fusion Res. 5, 428 (2002).

${ }^{10}$ T. Tamano, Phys. Plasmas 2, 2321 (1995). 